\title{
Lumbar artery branch injury secondary to ureteroscopic manipulation
}

\author{
Michael B. Rothberg, MD; ${ }^{*}$ Manoj Monga, MD; ${ }^{\dagger}$ Mantu Gupta, $M D^{*}$ \\ *Columbia University Medical Center, New York, NY; † Glickman Urological and Kidney Institute, The Cleveland Clinic, Cleveland, $\mathrm{OH}$
}

Cite as: Can Urol Assoc J 2014;8(5-6):449-50. http://dx.doi.org/10.5489/cuaj.1972 Published online June 19, 2014.

\section{Abstract}

latrogenic vascular injury in ureteroscopy is relatively uncommon and typically presents as a subcapsular hematoma. We report the case of an injury to a branch of the lumbar artery secondary to ureteral trauma during ureteroscopy. In the immediate postoperative period, the patient became hypotensive and tachycardic, prompting a workup that revealed an active hemorrhage. The patient then underwent subsequent angiogram and successful coil embolization.

\section{Case report}

Iatrogenic vascular injury in ureteroscopy is relatively uncommon and typically presents as a subcapsular hematoma. We report the case of a 58-year-old woman who underwent ureteroscopy, laser lithotripsy, basket extraction, and stent placement for an impacted stone in her right proximal ureter and multiple renal collecting system stones at an outside hospital. While performing ureteroscopy, severe edema was noted and laser lithotripsy was used in situ to free the impacted stone. The operative time was 63 minutes. In the immediate postoperative period, the patient became hypotensive, tachycardic, and hypokalemic with a rapidly decreasing hematocrit, requiring an intravenous infusion of $\mathrm{KCl}$ and a transfusion of 2 units of packed red blood cells for stabilization. A computed tomography scan with intravenous contrast of the abdomen was obtained and the interventional radiology service was consulted to confirm the presence of a severe, intra-abdominal hemorrhage originating from a branch of the right L1 lumbar artery. The patient was then taken to the interventional suite for urgent embolization of the injured vessel, classified as a Clavien IIIb complication.

Using fluoroscopic guidance, we accessed the injured lumbar artery branch via the right common femoral artery.
An angiogram was performed on the L1 lumbar artery branch to identify the area of active extravasation (Fig. 1a), which was then embolized with two $2 \times 2-\mathrm{mm}$ straight fibered platinum coils and a $2 \times 5-\mathrm{mm}$ Figure $8-18$ fibered platinum coil. Successful coil embolization was confirmed by a completion angiogram (Fig. 1b).

The postoperative course was unremarkable with stabilization of hematocrit; she was discharged on postoperative day 2 . The patient was then seen at our institution and was found to have retained stones in the ureter and kidney with an indwelling stent. She then underwent successful ureteroscopy, laser lithotripsy, and stone extraction without any further complications or vascular symptoms. During the procedure, the likely area of prior injury was noted as a healed area of mucosa in the medial wall of the proximal ureter (Fig. 2).

\section{Discussion}

To our knowledge, there is no literature detailing an iatrogenic injury to a branch of the lumbar artery secondary to ureteroscopic manipulation. It is believed that this vascular injury may have occurred secondary to the use of laser energy on a stone impacted on the ureteral wall; this injury could be prevented by mobilizing the stone into the kidney prior to performing laser lithotripsy. This case report presents the possibility of a major vascular injury and severe hemorrhage in the setting of ureteroscopy.

Vascular injuries have occurred during ureteroscopy; several case reports and case series describe patients with subcapsular renal hematomas (SRH) following ureteroscopy with an incidence of $0.4 \% .^{1-3}$ While both a lumbar artery branch rupture and a SRH can present with unexplained decreasing hematocrit or flank pain, the timing of onset of the symptoms may vary depending on the nature of the injury. Our patient presented with hypotension, tachycardia, and a decreasing hematocrit in the immediate postoperative period due to the severe nature of the hemorrhage; on the 


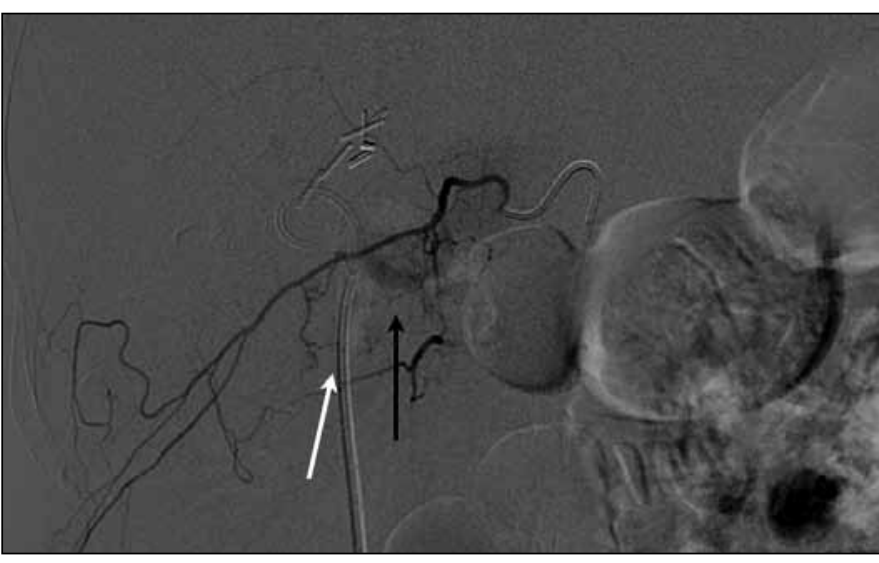

Fig. 1a. Contrast angiogram of lumbar artery L1 branch demonstrating location of vessel injury (white arrow) near the proximal ureter and subsequent extravasation of contrast (black arrow).

contrary, in a case series by Bai and colleagues, patients with a SRH presented with symptoms anywhere between 4 to 10 hours postoperatively. ${ }^{2}$

\section{Conclusion}

Urologists should be aware of the possibility of this complication in the immediate postoperative period and realize the necessity to urgently manage such a condition with vessel embolization.

Competing interests: Dr. Rothberg, Dr. Monga and Dr. Gupta all declare no competing financial or personal interests.

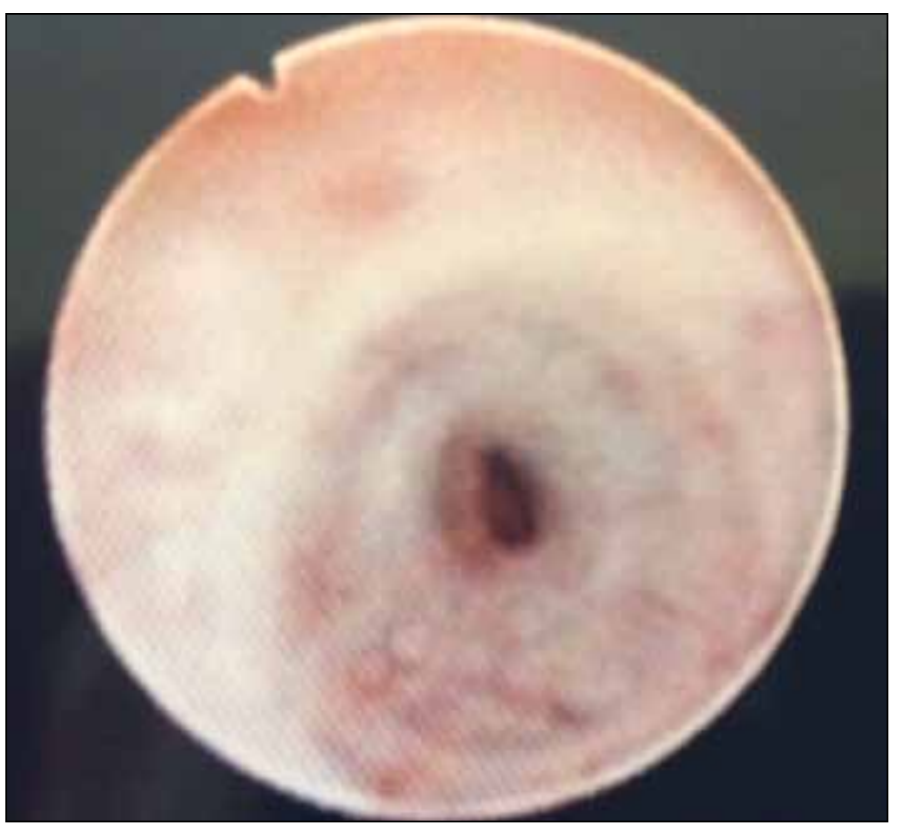

Fig. 2. Image from ureteroscope demonstrating area of healed mucosa in the proximal ureter.

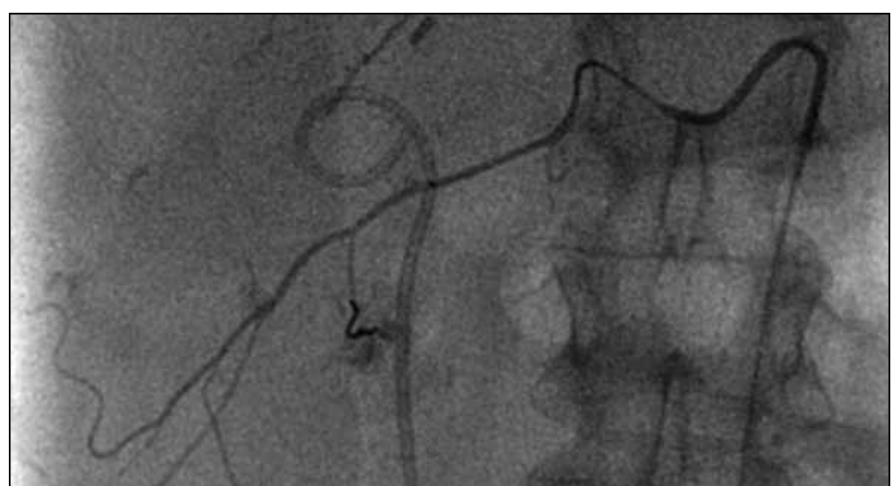

Fig. 1b. Contrast angiogram of lumbar artery L1 branch following coil embolization at site of vascular injury.

This paper has been peer-reviewed.

\section{References}

1. Chiu PK, Chan C, Ma W, et al. Subcapsular hematoma after ureteroscopy and laser lithotripsy. J Endourol 2013;27:1115-9. http://dx.doi.org/10.1089/end.2013.0128

2. Bai J, Li C, Wang S, et al. Subcapsular renal hematoma after holmium:yttrium-aluminum-garnet laser ureterolithotripsy. BJU Int 2011;109:1230-4. http://dx.doi.org/10.1111/j.1464-410X.2011.10490.x

3. Duffey BG, Lee JY, Monga M. Perinephric hematoma following ureteroscopy and holmium laser lithotripsy. Open Urol Nephrol J 2008;1:36-7. http://dx.doi.org/10.2174/1874303X00801010036

Correspondence: Dr. Mantu Gupta, Columbia University Medical Center, Department of Urology, 161

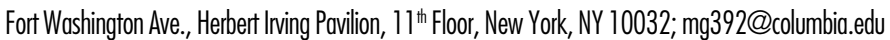

